\title{
Muskuwaari, immigration et mutations spatio- agricoles en pays guiziga (Extrême-Nord Cameroun)
}

Felix Watang Zieba

\section{(2) OpenEdition Journals}

Édition électronique

URL : http://journals.openedition.org/cdg/2969

DOI : 10.4000/cdg.2969

ISSN : 2107-7266

Éditeur

UMR 245 - CESSMA

Référence électronique

Felix Watang Zieba, « Muskuwaari, immigration et mutations spatio-agricoles en pays guiziga

(Extrême-Nord Cameroun) », Carnets de géographes [En ligne], 2 | 2011, mis en ligne le 02 mars 2011, consulté le 07 mai 2019. URL : http://journals.openedition.org/cdg/2969; DOI : 10.4000/cdg.2969

Ce document a été généré automatiquement le 7 mai 2019.

\section{(c) (†) $\ominus$}

La revue Carnets de géographes est mise à disposition selon les termes de la Licence Creative Commons Attribution - Pas d'Utilisation Commerciale - Pas de Modification 4.0 International. 


\title{
Muskuwaari, immigration et mutations spatio-agricoles en pays guiziga (Extrême-Nord Cameroun)
}

\author{
Felix Watang Zieba
}

Cette étude qui porte sur les mutations spatio-agricoles en pays guiziga dans la région de l'Extrême-Nord Cameroun ( $\mathrm{N} 10^{\circ}-13^{\circ}$ et E13 $\left.13^{\circ} 15^{\prime}-15^{\circ} 45^{\prime}\right)$ a pour objectif de comprendre la dynamique actuelle de cet espace longtemps qualifié de zone d'émigration.

2 Situé entre les latitudes $10^{\circ}$ et $10^{\circ} 30^{\prime} \mathrm{N}$ et les longitudes $14^{\circ}$ et $14^{\circ} 30^{\prime} \mathrm{E}$, le pays guiziga est l'un des vieux bassins de peuplement des plaines de l'Extrême-Nord Cameroun. Il s'étend à la fois sur les départements du Mayo Kani et du Diamaré. Ses densités moyennes (30hts/ $\mathrm{km}^{2}$ ) justifient la disponibilité des vertisols encore inexploités et de plus en plus convoités par des migrants venus du pays toupouri situé dans la vallée du Logone plus au sud de la même région. 
Figure 1. Localisation de la zone d'étude.

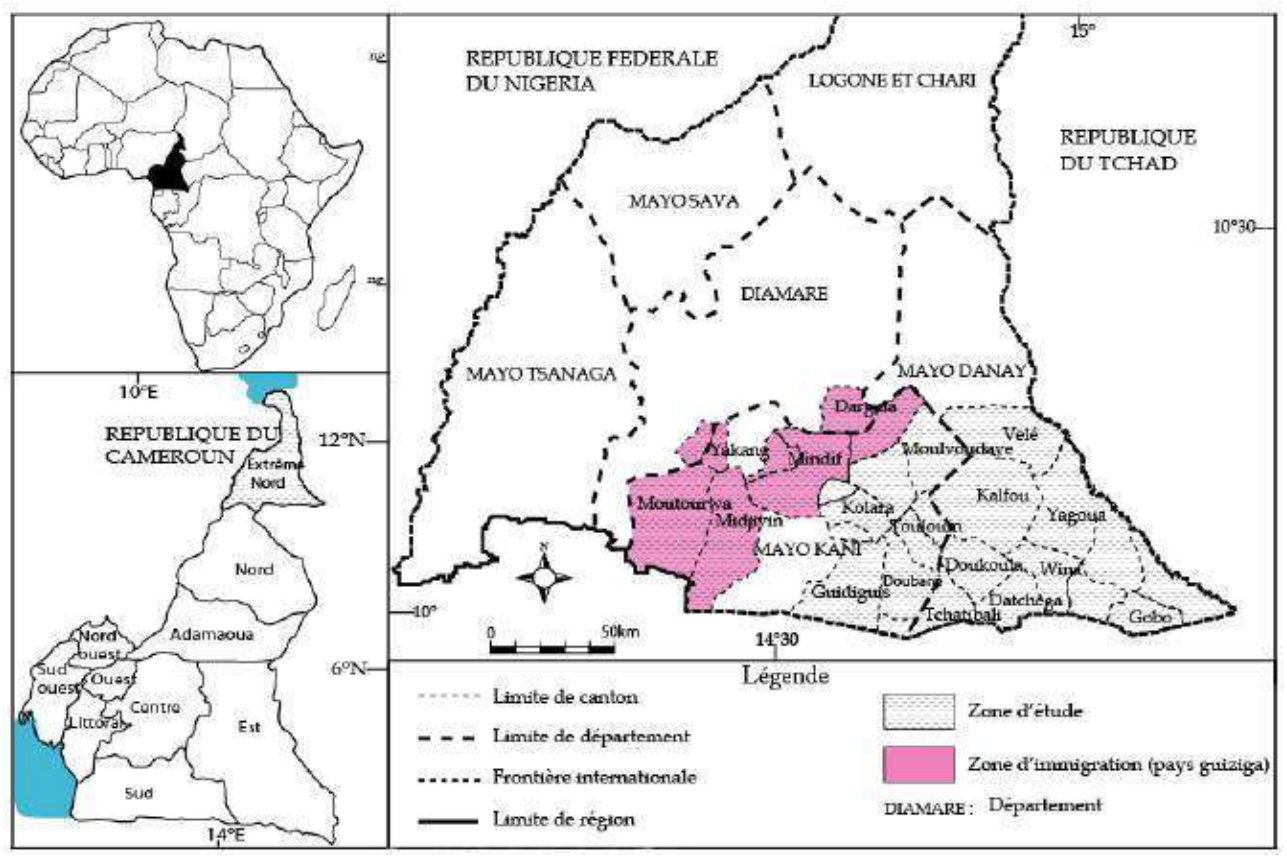

Source fond de carte : Atlas Ext. Nord (2000) Réalisé par Watang Ziéba en mars 2007 et révisé en novembre 2008

3 Le pays guiziga a constitué pendant près de quarante ans une zone de départ majeure pour $13 \%$ de la population migrante dans la plaine de la Bénoué située dans la région du Nord aux conditions climatiques plutôt favorables à l'agriculture. Cependant, cette même zone (pays guiziga) connaît depuis le début des années 1990, un regain de dynamisme spatio-agricole non moins important. On y constate le défrichement des derniers espaces inoccupés et l'occupation des zones interstitielles entre les différents terroirs guiziga, par des immigrants originaires de la vallée du Logone en quête d'espace de culture de sorgho de contre saison (muskuwaari). Si l'ampleur de ce mouvement migratoire récent est relativement faible par rapport à celle des autres courants migratoires encadrés au Nord Cameroun (migrations vers la vallée de la Bénoué), il faut souligner qu'il s'agit de l'installation de plus de 400 familles de migrants, dans plus de 20 terroirs situés en pays guiziga, répartis dans 4 lamidats ${ }^{1}$ (Moutourwa, Midjivin, Mindif, Maroua), 4 arrondissements (Maroua, Moutourwa, Mindif, Dargala), bref dans le dernier espace ethnique homogène guiziga.

est question ici de mettre en exergue cette nouvelle orientation de la mobilité dans la région de l'Extrême-Nord Cameroun tout en redéfinissant tout le système migratoire ruro-rural du Nord Cameroun avant d'analyser la relation conséquente entre ces nouvelles mobilités spatiales de la population et les mutations spatio-agricoles actuelles en pays guiziga.

5 L'analyse des informations qui résultent de l'observation de terrain (enquêtes, entretiens, levés au GPS, prises de vue effectués entre 2003 et 2007) et de l'exploration des documents (archives et ouvrages scientifiques) montrent que les mutations agricoles, foncières et territoriales sont en relation avec les mobilités spatiales récentes observées à l'intérieur de cette région. Elles se caractérisent surtout par une orientation sud/nord. Les mutations agricoles sont considérables à l'échelle de l'exploitation, d'un terroir 
d'immigration à un autre. Ceci tant au niveau de l'évolution de la taille des parcelles que de la production du muskuwaari. Quant aux mutations foncières, elles sont visibles à travers la transformation des modes d'accès à la terre et les stratégies mises en place par les acteurs immigrants et Guiziga. L'exemple des terroirs d'immigration témoins de Barawa Laddé, de Foulou et de Mobono méthodiquement choisis, illustre parfaitement ce point de vue.

Par ailleurs, l'échelle supa-locale nous permet de voir une construction de territoires agricoles par les migrants au-delà des limites centenaires des lamidats. C'est le cas du petit terroir de Mobono qui s'est construit sur deux lamidats (Moutourwa et Mindif). Les frontières des lamidats sont alors de moins en moins considérées comme des obstacles à l'extension des espaces agricoles. On note aussi une recomposition du territoire d'immigration à travers l'éclatement de l'un des derniers espaces ethniques homogènes de la région de l'Extrême-Nord Cameroun.

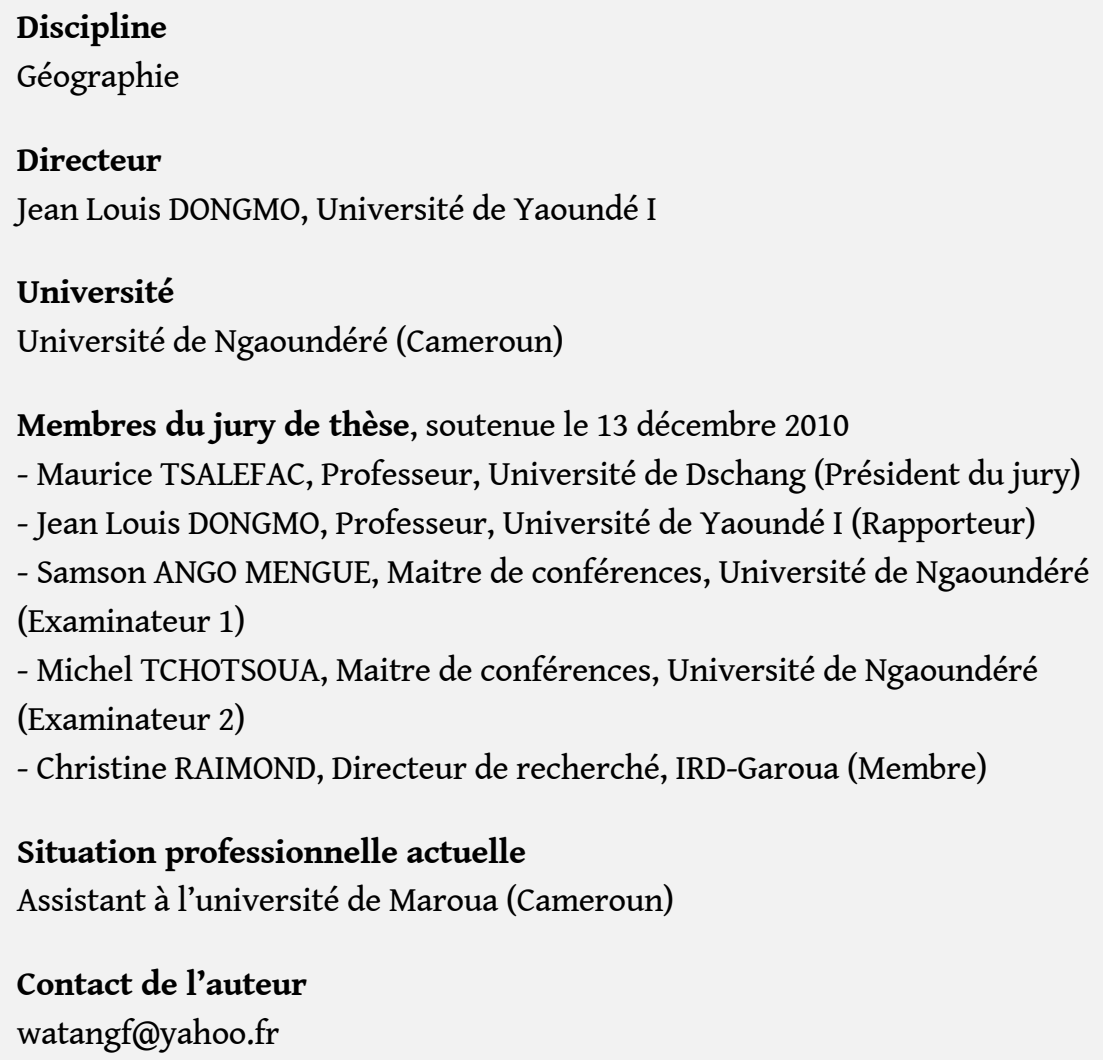

\section{NOTES}

1. Unité territoriale traditionnelle précoloniale qui a à sa tête un chef traditionnel ou lamido. 
INDEX

Thèmes : Carnets de soutenances 